\title{
Measurement of Inner Bark and Leaf Osmolality
}

\section{Paljakka, Teemu}

Humana press

2019

Paljakka , T , Lintunen , A , Salmon , Y \& Hölttä , T 2019 , Measurement of Inner Bark and Leaf Osmolality . in J Liesche (ed.), Phloem : Methods and Protocols . Methods in Molecular Biology , vol. 2014 , Humana press , pp. 135-142 . https://doi.org/10.1007/978-1-4939-9562-2_11

http://hdl.handle.net/10138/316139

https://doi.org/10.1007/978-1-4939-9562-2_11

unspecified

acceptedVersion

Downloaded from Helda, University of Helsinki institutional repository.

This is an electronic reprint of the original article.

This reprint may differ from the original in pagination and typographic detail.

Please cite the original version. 


\section{Measurement of Inner Bark and Leaf Osmolality}

Teemu Paljakka $1 \square$

Email teemu.paljakka@helsinki.fi

Anna Lintunen 1,2

Yann Salmon 1,2

Teemu Hölttä 1 AQ1

1 Faculty of Agriculture and Forestry, Institute for Atmospheric and Earth System Research/Forest Sciences, University of Helsinki, Helsinki, Finland

2 Faculty of Science, Institute for Atmospheric and Earth System Research/Physics, University of Helsinki, Helsinki, Finland

\section{Abstract}

Sugar transport in the phloem is driven by turgor pressure gradients which are created by osmotic gradients resulting from sugars loaded to the phloem at the source tissue and unloaded at the sink tissue. Therefore, osmolality is a key parameter that can be used to evaluate sugar status and get an indication of the driving force for phloem transport. Here we describe how osmotic concentration measurements from inner bark (practically, the phloem) and needles of trees can be measured. This protocol presents the procedure used by Lintunen et al. (Front Plant Sci 7:726, 2016) and Paljakka et al. (Plant Cell Environ 40:2160-2173, 2017), extended by practical advice and discussion of potential errors and caveats. We describe how to implement this procedure for gymnosperm as well as angiosperm trees. This method uses mechanical sap extraction with a centrifuge from inner bark and leaf samples, which have gone through a deep freeze treatment and thawing. The osmotic potential of these samples is then analyzed with a freezing point or vapor pressure osmometer. The aim of these measurements is to study the spatial and temporal dynamics of phloem function.

$\mathrm{AQ2}$

\section{Key words}

Phloem

Osmolality

Osmotic potential

Water potential

Conifer

Method description
Note: This is the final author's version of manuscript of the following book chapter:

\author{
"Paljakka, T., Lintunen, A., Salmon, Y., \& Hölttä, T. (2019). \\ Measurement of Inner Bark and Leaf Osmolality. In Phloem (pp. 135-142). \\ Humana, New York, NY.",
}

which has been published in final form at

[https://doi.org/10.1007/978-1-4939-9562-2_11].

\section{Introduction}

Osmolality is an important quantity in plant function since it is required to draw in water which is needed to create the turgor pressure that sustains metabolic activity. Furthermore, solute transport in phloem is driven by turgor pressure gradients which, in turn, are created by osmotic gradients resulting from sugars loaded to the phloem from the source tissue and unloaded in the sink tissue $[1,2]$. The phloem water potential is the sum of turgor pressure and osmotic potential. Osmolality is linearly proportional to osmotic potential and independent of temperature as it is a measure of mass and not volume [3]. Osmolality of the inner bark or leaves is a rather robust measure of the overall contribution of osmotically active solutes and water in the living cells of these tissues. Interpreting the phloem solute content based on more detailed sugar concentration analyses have challenges in terms of comparing absolute values among different methods and laboratories [4]. Moreover, aiming to measure the osmolality of the conducting phloem only is more 
challenging than measuring the osmolality of the whole inner bark from the sampling point of view [5] as the conducting phloem is a small fraction of the inner bark, and methods for separating the tissues are

challenging under field conditions. The phloem is also highly sensitive to damage caused by sampling, e.g., because of pressure release from the sieve cells (e.g., $[6,7])$. Together these issues create a high risk of error when interpreting experimental data with regard to the dynamics of phloem transport.

AQ3

The sampling and sap extraction procedure described in this chapter estimates the in situ osmolality of inner bark (e.g., $[1,5,8,9])$, including tissues from cambium to cork cambium, and the in situ osmolality of leaves in conifers (e.g., $[10,11])$ and angiosperms (e.g., $[8,12])$. This procedure relies on the assumption that solutes from the symplast are released to the apoplast during deep freezing and thawing followed by mechanical sap extraction. The sampled sap osmolality is a measure of the quantity of all the dissolved substances such as sucrose, monosaccharides, alcohols, potassium, and other osmotically active solutes in a given amount of solution. Water potential and tissue properties change along the stem, and between branches and stem, with larger proportion of sieve cells toward the stem base [3, 13]. Therefore, differences in solution mixtures are expected at different tree parts. Note that the procedure described here is not targeted to measure the osmolality of the phloem sap transported in the sieve tubes. Instead, the osmolality of the whole inner bark or leaves is measured. Estimates of sap solute content (see Note 1) and turgor pressure (see Note 2) are possible with osmolality measurements when underlying assumptions and limitations are taken into consideration.

\section{Materials}

Materials in this section are described as in the studies of Lintunen et al. [14] and Paljakka et al. [15], and the measurement details with the vapor pressure osmometer as in Salmon et al. [16].

\subsection{Sampling}

1. Scalpel or knife for collecting inner bark samples.

2. Freezing-tolerant cryo tubes (e.g., $5 \mathrm{~mL}$ cryo tube).

3. Dry shipper, cool box with dry ice, or liquid nitrogen for transporting samples, and for the temporal deep freezing treatment to brake the cell walls allowing the cell sap to be extracted.

4. Deep freezer set to $-80{ }^{\circ} \mathrm{C}$ or liquid nitrogen tank for preserving the samples for longer times.

\subsection{Sap Extraction and Measurement of Osmolality}

1. Microcentrifuge; equipped with 24-place rotor for 1.5 or $2 \mathrm{~mL}$ tubes, capable of a speed of $14,000 \times g$.

2. Freezing point osmometer (Osmomat 030 cryoscopic osmometer, Gonotec, Berlin, DE) or vapor pressure osmometer (Vapro 5520, Wescor Inc., Logan, UT, USA).

3. Distilled water and $300 \mathrm{~mol} \mathrm{~kg}^{-1} \mathrm{NaCl}$ solution for calibration of the freezing point osmometer. For higher sample concentrations than $700 \mathrm{~mol} \mathrm{~kg}^{-1}$, additional calibration solution with higher concentration, e.g., $\mathrm{NaCl}$ solution of $850 \mathrm{~mol} \mathrm{~kg}^{-1}$ (three-point calibration), should be included.

4. Pipettes with disposable tips for moving small amounts of liquid sample (e.g., $50 \mu \mathrm{mL})$.

5. Tubes for the centrifuge with a liquid separating column; for example, cellulose acetate filtered centrifuge tubes or silica-based membrane columns (as found, for example, in DNA miniprep kit) can be used. 
6. Measurement vessels for the freezing point osmometer, or paper sample discs when using the vapor pressure osmometer.

7. Scalpel and forceps.

8. Distilled water and ethanol solution for cleaning of tools during sample preparation.

\section{Methods}

\subsection{Sampling in the Field}

When planning sample positions and number of replicates, it should be taken into consideration that osmolality values vary spatially and temporally (see Note 3 ). In addition, the amount of damage caused to the tree due to sampling should be considered if the tree is monitored for longer periods.

1. Remove cork before collecting the inner bark, e.g., with knife or scalpel (see Note 4).

2. Collect the inner bark sample, typically a piece of ca. $2 \mathrm{~cm}^{2}$ (see Note 5). A scalpel has been found to be a good tool for cutting the sample borders in the bark. Thereafter, the collection could be made with a blunt knife, which allows better extraction of bark without cutting into the underlying xylem. Sample collection from branches can be carried out the same way with similar sample size as in the stem, or with smaller branches by first cutting the branch and then collecting the bark sample by peeling it off the wood surface.

3. Pick leaf samples by hand if the canopy is within reach (see Note 6). Five pairs of $P$. sylvestris needles is often more than enough to extract the required liquid sample (ca. 50-100 $\mu \mathrm{mL}$ ). Often, one leaf is enough to obtain sufficient amount of sample in angiosperms, but this depends on the species.

\subsection{Storage}

1. After collection, immediately place the sample into a sealed cryo tube, and place the tube into dry shipper or dry ice to avoid evaporation and changes in solute composition.

2. Transfer samples to deep freeze, either in $\mathrm{a}-80{ }^{\circ} \mathrm{C}$ freezer or in a liquid nitrogen tank, if sample extraction is not executed immediately after deep freeze treatment of samples (see Note 7).

\subsection{Sample Preparation}

1. Thaw samples inside the closed tubes at room temperature for ca. $15 \mathrm{~min}$.

2. Before placing bark samples to centrifuge tubes containing the liquid separating column, cut the end of the inner bark sample facing the filter with a scalpel to ease the collection of sap (Fig. 1a). Filtered centrifuge tubes are only used to separate the sap, including the dissolved compounds, from the solid parts of the plant tissue. Needles can be cut in half and the cut side set downward against the filter. Angiosperm leaves can be cut perpendicular to the main leaf vein, in, e.g., $5 \mathrm{~mm}$ wide slices, and rolled inside the tubes with the cut side downward against the filter. To avoid crosscontamination between samples, sampling equipment, especially scalpel blades, should be cleaned between preparations, e.g., with ethanol solution.

3. Centrifuge at $14,000 \times g$ for $10 \mathrm{~min}$ for efficient liquid extraction from the sample $[9,14,15]$. 
Inner bark piece, with the filter-facing end toward the tube, to be set inside a filtered centrifuge tube (a) and extracted sap ready to be measured in osmometer (b)

\section{(a)}

\subsection{Osmolality Measurement with Freezing Point Osmometer}

Devices for measuring osmolality differ. One option is to use freezing point osmometer where osmolality is defined with the equilibrium freezing point of a solution $[14,15]$.

1. Calibrate device following the device manual. In the studies of Lintunen et al. [14] and Paljakka et al. [15], the osmometer was calibrated with a two-point calibration with distilled water and $0.300 \mathrm{~mol} \mathrm{~kg}^{-1}$ solutions of $\mathrm{NaCl}$. With this calibration setup $\pm 1 \%$ variance from linearity can be obtained between concentrations 0 and $700 \mathrm{~mol} \mathrm{~kg}^{-1}$.

2. Transfer the sap extracted from the tissue to the measurement vessels designed for osmolality measurement using a pipet (see Note 8). The sap sample should not include air bubbles or visible impurities since they can interfere with the measurement (see Note 9). Similar liquid sample size is recommended for consistent measurements.

3. Place the vessel in the instrument with the instrument sensor submerged in the sampled sap (Fig. 1b).

4. Measure osmolality of the sample according to the instrument manufacturer's instructions. 
5. Write down the measured osmolality value, which is given in the unit mol $\mathrm{kg}^{-1}$.

6. Rinse the sensor of the osmometer with milli-Q or distilled water, and wipe with lens paper between each measurement.

\subsection{Osmolality Measurement with Vapor Pressure Osmometer}

1. Calibrate device following the device manual.

2. Open the sample chamber and, using forceps, place a single sample disc in the disc holder depression.

3. Carefully, take precisely $10 \pm 1 \mu \mathrm{L}$ from the sample using a pipet with disposable tips (change tips after each sample) and place them on the disc.

4. Close the sample chamber and wait for reading.

5. Write down the measured osmolality value.

6. Remove the disc after the measurements and clean the sample holder with a lint-free tissue.

\section{Notes}

1. To estimate the amount of osmotically active solutes in the sample $[5,15,17]$, one should measure also the relative water content [18], for example, from an adjacent bark piece. Then in situ osmolality values can be used in the calculation of the osmotically active solutes (osmolality at full saturation) as follows [17].

$$
\mathrm{osMOL}_{\text {full saturation }}=\mathrm{osMOL}_{\text {in situ }} \times \mathrm{RWC}
$$

where osMOL ${ }_{\text {in situ }}$ is the in situ osmolality and RWC is relative water content.

2. Assuming water potential equilibrium between the xylem and inner bark, the inner bark turgor pressure can be estimated by measuring simultaneously the xylem water potential at the same position when collecting the samples for osmolality $[3,8,19]$. From leaves and branches, this can be conducted easily with a pressure chamber.

3. With the procedure described in this chapter, osmolality in situ has been observed to range from ca. $0.4 \mathrm{~mol} \mathrm{~kg}^{-1}$ in the stem base up to ca. $0.7 \mathrm{~mol} \mathrm{~kg}^{-1}$ in the inner bark of branches, and up to around $0.8 \mathrm{~mol} \mathrm{~kg}^{-1}$ in the needles in P. sylvestris and Picea abies $[14,15]$ in boreal conditions. These values are similar to previously reported osmolality values in the needles and upper stem of $P$. sylvestris and $P$. abies (e.g., $[5,11])$. The daily variation of osmolality can be more than $0.1 \mathrm{~mol} \mathrm{~kg}^{-1}$ depending on the sample location in a tree (Fig. 2). The mean inner bark osmolality of branches has been reported to be ca. $0.5-0.7 \mathrm{~mol} \mathrm{~kg}^{-1}$ in Populus tremula and Betula pendula in moist and dry sites around the Europe [14]. Signs of seasonal differences in osmolality values have been found at least in conifer needles [15]. Additionally, environmental variables, such as VPD, light, soil water content, and temperature, can be expected to affect osmolality through their effects on source and sink strength and xylem water potential.

4. Removing the cork entirely from a detached bark piece is sometimes challenging, especially in the lower parts of the conifer stem. Additionally, the inner bark of birch branches have been found difficult to separate from the cork as the branches toward the tips are thin. However, small amounts 
of cork, with little water or osmotically active solutes, can be expected to affect the osmolality of a sampled liquid only marginally.

5. The sample size depends ultimately on the minimum sample size the osmometer requires for measuring, e.g., $15 \mu \mathrm{L}$ of liquid sample [14, 15]. Salmon et al. [16] used a vapor pressure osmometer for which precisely $10 \mu \mathrm{L}$ samples are needed. In theory, sample size should not matter as we assume a mixture of solutes per mass. False readings (i.e., instrument returning readings of too high values) have been encountered when the amount of extracted sap is too small (less than the minimum sample amount set by the manufacturer). Furthermore, small samples are more prone to evaporation and thus to changes in solute concentration. The amount of liquid needed determines the size of the bark sample that needs to be collected. This will be different in different locations, tree species, and environmental conditions. For example, the minimum bark sample size of $P$. sylvestris at our site in Southern Finland is ca. $2 \mathrm{~cm}^{2}$ depending on the sample position in the tree. A piece of this size yields ca. $30-50 \mu \mathrm{L}$ of sap. The inner bark in the stem of $P$. sylvestris is thicker in the younger stem than at the stem base. Therefore, a smaller piece (e.g., $0.5 \mathrm{~cm}$ width $\times 1.5 \mathrm{~cm}$ vertical length) may be enough at higher positions in the stem to acquire the required amount of sample. However, the needed sample size might be larger in dry conditions and in dry sites, and this should be tested before the collection. For very dry samples ( WP $<-2.5 \mathrm{MPa}$ for P. sylvestris) it might be impossible to extract enough liquid with the centrifugation.

6. Excess water should be removed from the samples to avoid dilution of the extracted sap if the sampling has been conducted in rainy conditions.

7. Freezing in very cold temperatures (e.g., $-80^{\circ} \mathrm{C}$ ) and thawing is expected to brake most of the membranes of the living cells releasing the solutes into the apoplast.

8. After the liquid is extracted by centrifugation, great care has to be taken to prevent evaporation from the small sample volume. The sample number at each set of analyses is recommended to be kept sufficiently small. Additionally, evaporation of the samples should be avoided by closing tubes with lids, and by carrying out the measurement procedure from thawing to osmolality measurement as quickly as possible.

9. The freezing point osmometer may sometimes return "spontaneous crystallization" because of impurities in the solution that affect the measurement of the solution's freezing point. Because of this, more than one sample should be obtained from each location.

Fig. 2

Example set of in situ osmolality values in needles, and the inner bark of branches, upper stem and stem base in P. sylvestris during 2 days in September in Southern Finland. Data adapted from Paljakka et al. [15] 


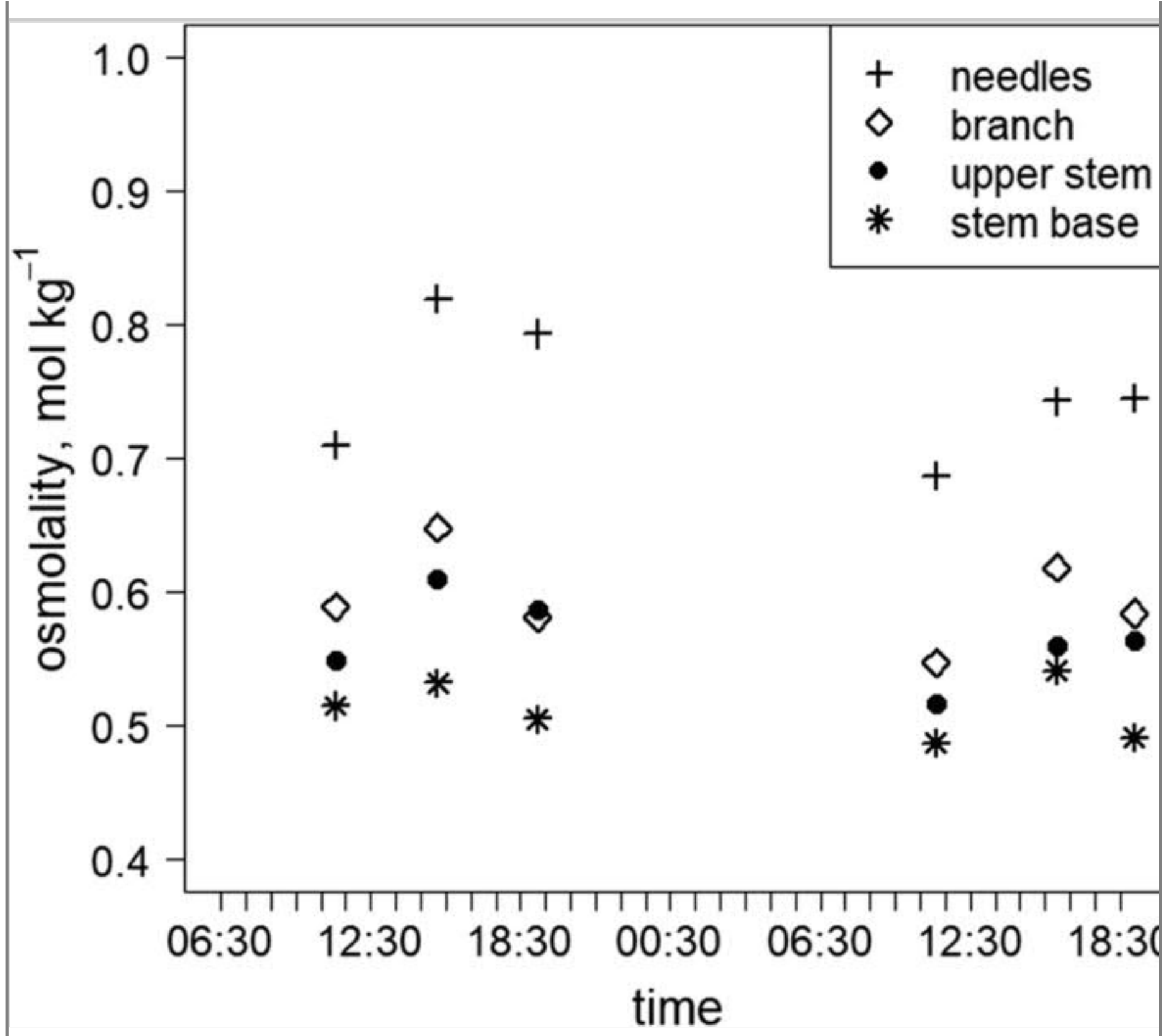

\section{References}

1. Münch E (1930) Die Stoffbewegungen in der Pflanze. Gustav Fischer, Jena, pp 124-146

2. Liesche J, Schulz A (2018) Phloem transport in gymnosperms: a question of pressure and resistance. Curr Opin Plant Biol 43:36-42

3. Nobel PS (2005) Physiochemical and environmental plant physiology, 3rd edn. WH Freeman and Company, New York

4. Quentin AG, Pinkard EA, Ryan MG, Tissue DT, Baggett LS, Adams HD et al (2015) Non-structural carbohydrates in woody plants compared among laboratories. Tree Physiol 35(11):1146-1165

5. Rosner S, Baier P, Kikuta SB (2001) Osmotic potential of Norway spruce [Picea abies (L.) Karst.] secondary phloem in relation to anatomy. Trees 15(8):472-482

6. Sovonick-Dunford S, Lee DR, Zimmermann MH (1981) Direct and indirect measurements of phloem turgor pressure in white ash. Plant Physiol 68(1):121-126 
7. van Bel AJ (2003) The phloem, a miracle of ingenuity. Plant Cell Environ 26(1):125-149

8. Kaufmann MR, Kramer PJ (1967) Phloem water relations and translocation. Plant Physiol 42(2):191-194

9. Devaux M, Ghashghaie J, Bert D, Lambrot C, Gessler A, Bathellier C et al (2009) Carbon stable isotope ratio of phloem sugars in mature pine trees throughout the growing season: comparison of two extraction methods. Rapid Commun Mass Spectrom 23(16):2511-2518

10. Huttunen S, Kärenlampi L, Kolari K (1981) Changes in osmotic potential and some related physiological variables in needles of polluted Norway spruce (Picea abies). In: Annales Botanici Fennici. Finnish Botanical Publishing Board, New York, NY, pp 63-71

11. Irvine J, Perks MP, Magnani F, Grace J (1998) The response of Pinus sylvestris to drought: stomatal control of transpiration and hydraulic conductance. Tree Physiol 18(6):393-402

12. Scholander PF, Hammel HT, Hemmingsen EA, Bradstreet ED (1964) Hydrostatic pressure and osmotic potential in leaves of mangroves and some other plants. Proc Natl Acad Sci U S A 52(1):119-125

13. Jyske T, Hölttä T (2015) Comparison of phloem and xylem hydraulic architecture in Picea abies stems. New Phytol 205(1):102-115

14. Lintunen A, Paljakka T, Jyske T, Peltoniemi M, Sterck F, Von Arx G et al (2016) Osmolality and nonstructural carbohydrate composition in the secondary phloem of trees across a latitudinal gradient in Europe. Front Plant Sci 7:726. https://doi.org/10.3389/fpls.2016.00726

15. Paljakka T, Jyske T, Lintunen A, Aaltonen H, Nikinmaa E, Hölttä T (2017) Gradients and dynamics of inner bark and needle osmotic potentials in Scots pine (Pinus sylvestris L.) and Norway spruce (Picea abies (L.) Karst). Plant Cell Environ 40(10):2160-2173. https://doi.org/10.1111/pce.13017

16. Salmon Y, Torres-Ruiz JM, Poyatos R, Martinez-Vilalta J, Meir P, Cochard H, Mencuccini M (2015) Balancing the risks of hydraulic failure and carbon starvation: a twig scale analysis in declining Scots pine. Plant Cell Environ 38(12):2575-2588

17. Takami S, Turner NC, Rawson HM (1981) Leaf expansion of four sunflower (Helianthus annuus L) cultivars in relation to water deficits. I. Patterns during plant development. Plant Cell Environ 4(5):399407

18. Smart RE, Bingham GE (1974) Rapid estimates of relative water content. Plant Physiol 53(2):258-260

19. Hölttä T, Vesala T, Sevanto S, Perämäki M, Nikinmaa E (2006) Modeling xylem and phloem water flows in trees according to cohesion theory and Münch hypothesis. Trees 20(1):67-78 